OPEN ACCESS

Edited by:

Lin-Hua Jiang,

University of Leeds, United Kingdom

Reviewed by:

Juan C. Saez,

Pontificia Universidad Católica

de Chile, Chile

Yong Tang,

Chengdu University of Traditional

Chinese Medicine, China

*Correspondence:

Jorge Fuentealba

jorgefuentealba@udec.cl

Specialty section:

This article was submitted to Integrative Physiology, a section of the journa

Frontiers in Physiology

Received: 14 November 2021

Accepted: 12 January 2022

Published: 23 February 2022

Citation:

Castillo C, Saez-Orellana F

Godoy PA and Fuentealba J (2022)

Microglial Activation Modulated by

P2X4R in Ischemia

and Repercussions in Alzheimer's

Disease. Front. Physiol. 13:814999.

doi: 10.3389/fphys.2022.814999

\section{Microglial Activation Modulated by P2X4R in Ischemia and Repercussions in Alzheimer's Disease}

\section{Carolina Castillo, Francisco Saez-Orellana, Pamela Andrea Godoy and Jorge Fuentealba*}

Laboratory of Screening of Neuroactive Compounds, Department of Physiology, School of Biological Sciences, Universidad de Concepción, Concepción, Chile

There are over 80 million people currently living who have had a stroke. The ischemic injury in the brain starts a cascade of events that lead to neuronal death, inducing neurodegeneration which could lead to Alzheimer's disease (AD). Cerebrovascular diseases have been suggested to contribute to AD neuropathological changes, including brain atrophy and accumulation of abnormal proteins such as amyloid beta $(A \beta)$. In patients older than 60 years, the incidence of dementia a year after stroke was significantly increased. Nevertheless, the molecular links between stroke and dementia are not clearly understood but could be related to neuroinflammation. Considering that activated microglia has a central role, there are brain-resident innate immune cells and are about 10-15\% of glial cells in the adult brain. Their phagocytic activity is essential for synaptic homeostasis in different areas, such as the hippocampus. These cells polarize into phenotypes or subtypes: the pro-inflammatory M1 phenotype, or the immunosuppressive M2 phenotype. Phenotype M1 is induced by classical activation, where microglia secrete a high level of pro- inflammatory factors which can cause damage to the surrounding neuronal cells. Otherwise, M2 phenotype is the major effector cell with the potential to counteract pro-inflammatory reactions and promote repair genes expression. Moreover, after the classical activation, an antiinflammatory and a repair phase are initiated to achieve tissue homeostasis. Recently it has been described the concepts of homeostatic and reactive microglia and they had been related to major $A D$ risk, linking to a multifunctional microglial response to $A \beta$ plaques and pathophysiology markers related, such as intracellular increased calcium. The upregulation and increased activity of purinergic receptors activated by ADP/ATP, specially P2X4R, which has a high permeability to calcium and is mainly expressed in microglial cells, is observed in diseases related to neuroinflammation, such as neuropathic pain and stroke. Thus, P2X4R is associated with microglial 
activation. P2X4R activation drives microglia motility via the phosphatidylinositol-3kinase (PI3K)/Akt pathway. Also, these receptors are involved in inflammatory-mediated prostaglandin E2 (PGE2) production and induce a secretion and increase the expression of BDNF and TNF- $\alpha$ which could be a link between pathologies related to aging and neuroinflammation.

Keywords: P2X4R, microglial activation, ischemia, purinergic receptors, Alzheimer's disease

\section{INTRODUCTION}

Worldwide, there are over 80 million people currently living who have had a stroke (Lindsay et al., 2019). Over 13.7 million people have a stroke each year and 5.8 million dies because of it (Phipps and Cronin, 2020). Also, 116.4 million suffered permanent disabilities generated by stroke (Hachinski et al., 2019; Lindsay et al., 2019). It has been reported that 6 months post-stroke, around $35 \%$ of patients display depressive symptoms, $30 \%$ are unable to walk without assistance, and $26 \%$ are dependent on daily life activities (Lui and Nguyen, 2018). Stroke occurs when blood supply is interrupted, and there are two types: Ischemic and hemorrhagic (Vijayan et al., 2017). Ischemic strokes account for $85 \%$ of the total cases (Vijayan et al., 2017; Jayaraj et al., 2019; Lindsay et al., 2019). This type of stroke occurs when a blood vessel in the brain is occluded, causing a loss of the blood flow to an area of the brain (Randolph, 2016; Phipps and Cronin, 2020). The loss of blood flow causes the death of cells in the core of the injury, where the damage is irreversible, the surrounding area is called the penumbra and a loss of function is observed, but the damage is reversible (Phipps and Cronin, 2020). The ischemic injury in the brain starts a cascade of damaging cells events such as calcium dysregulation, increased reactive oxygen species (ROS) production, activation of caspases and calpain signaling to induce apoptosis and neuronal death, ultimately leading to neurodegeneration (Vijayan et al., 2017; Zhao et al., 2017; Castillo et al., 2018). The effects observed in patients depend on the brain region that is affected with supply oxygen and nutrients depletion (Vijayan et al., 2017). Due to this harmful process, some patients present neurological disorders. It has been estimated that stroke brings forward the onset of dementia by about 10 years (Vijayan et al., 2017; Lui and Nguyen, 2018). Current data estimates that dementia occurred in around $25 \%$ of people admitted to hospital with a stroke in the first year after this event (Hachinski et al., 2019). Furthermore, hazard ratios for dementia among patients who had strokes compared with the non-stroke population ranged from 2 to 8 , in different studies of severe stroke cases (Sala Frigerio et al., 2019). Alzheimer's Disease (AD) is the most common type of dementia, a neurological disorder characterized by neurodegeneration. From a biochemical perspective, AD is associated with synapse loss, synaptic dysfunction, mitochondrial structural and functional abnormalities, inflammatory responses, intracellular neurofibrillary tangles, and extracellular plaques (TrejoLopez et al., 2021). Clinically, AD is a multifactorial disease characterized by memory loss, multiple cognitive impairments, and progressive impairment of functional capacities. There are more than 100 pathological conditions that can be a risk factor for dementia, and stroke is the most common disease that can lead to this neurodegenerative disease (Vijayan and Reddy, 2016; Vijayan et al., 2017). AD and stroke have common risk factors, including hypertension, ROS increased levels, insulin resistance, type II diabetes, obesity, and hyperlipidemia (Hachinski et al., 2019; Sala Frigerio et al., 2019). Cerebrovascular diseases have been suggested to contribute to AD neuropathological changes including selective brain atrophy and abnormal accumulation of proteins such as amyloid beta peptide $(A \beta)$, which is described as the main toxic factor in AD (Hachinski et al., 2019).

\section{Aging Related Diseases: Stroke Incidence and Alzheimer's Disease}

Research linking stroke and dementia have been focused on common vascular risk factors, ameliorated by lifestyle activities or medication, nevertheless one of the most important risk factors is aging (Vijayan and Reddy, 2016; Vijayan et al., 2017). The risk of having a first-time stroke increases exponentially from about 30 per 100,000 individuals at 30-39 years of age, to about 2000-3000 per 100,000 at ages above 85 . Additionally, AD is mostly related to elderly patients, especially those over 80 years old (Mijajlović et al., 2017; Vijayan et al., 2017). Although, dementia due to stroke is not only aging-related, considering that this association has been reported in patients younger than 50 years old. Cognitive impairments occur in up to one-third of elderly patients with stroke (Lui and Nguyen, 2018). A mixed etiology of dementia and Vascular Dementia (VD) was thought to become more common with increasing age, but no clinical criteria for the diagnosis of dementia associated with stroke are available (Kluge et al., 2018). Stroke doubles the risk for dementia (post-stroke dementia), and approximately $30 \%$ of stroke patients develop cognitive dysfunction within 3 years (Kluge et al., 2018; Jayaraj et al., 2019; Lindsay et al., 2019). As a worrying statistic, stroke was the second cause of death and represented $9.1 \%$ of all deaths in 2017, and the third most common cause of deaths and disability combined (Delgado et al., 2010). It has been demonstrated that in patients older than 60 years, the incidence of dementia at 1 year after the stroke was $16 \%$, while the prevalence was $22 \%$ (Lavados et al., $2005,2021)$. The most frequent type of cognitive impairment was focal cognitive decline (Hachinski et al., 2019; Lavados et al., 2021). This study concludes that the risk of dementia after the first year in patients with severe strokes is very high, as it has been stated in several other studies (Lavados et al., 2021). Also, it has been mentioned the role of cerebrovascular disease, as a primary cause of cognitive impairment and to increase dementia risk caused by several factors, including $\mathrm{AD}$ or other neurodegenerative pathologies (Iadecola, 2013; Kalaria, 2018). 
During stroke and cerebrovascular diseases occurs hypoperfusion and blood brain barrer (BBB) disruption leading to oxidative stress and inducing tissue hypoxia by proteins extravasation. Hypoxia and oxidative stress activate pro-inflammatory pathways through kappa-light-chain-enhancer of activated B cells (NFkB) transcription, increasing cytokines and adhesion molecules in vascular cells, reactive astrocytes and activated microglia. That's promote uncoupling in the neurovascular unit, contributing to damaged vascular cells (Iadecola, 2013).

Also, from a molecular perspective, a brain ischemia induces a cascade of pathophysiological processes, which increase brain ischemia and stimulates the inflammatory process, free radical production, excitotoxicity, disruption of sodium and calcium influx, enzymatic changes, endothelin release, delayed coagulation, activation of platelets and leukocytes, and endothelial dysfunction. Otherwise, dementia syndromes, such as $\mathrm{AD}$, established after stroke were typically considered to be vascular in origin, and poststroke dementia might be the result of the effects of stroke and degenerative changes (Vijayan et al., 2017; Hachinski et al., 2019; Goulay et al., 2020). Nevertheless, the molecular links between stroke and dementia are not completely understood but are probably related to neuroinflammation (Kluge et al., 2018; Lui and Nguyen, 2018). Also, there are reported Longitudinal studies that have investigated the relation between inflammatory cytokines and post stroke dementia, suggesting interleukin 6, and interleukin 12 as predictors of post stroke cognitive impairment (Mijajlović et al., 2017).

Uncontrolled neuroinflammation, a hallmark of neurological disorders such as $\mathrm{AD}$ and stroke, may lead to neural dysfunction and aggravate disease progression. However, there are many questions raised in research linking stroke and dementia that are largely unanswered. Hence, it is important to understand early events of microglial cells activation, since they are the primary response involved in the inflammatory events during stroke or dementia such as AD.

\section{Role of Microglial Activation in Neuroinflammation}

Microglia are brain-resident innate immune cells with myeloid origin. At the resting state they are constantly sensing the environment to detect injury (Zhao et al., 2017). They account for about $10-15 \%$ of glial cells in the adult brain and their phagocytic activity is essential for synaptic homeostasis in different areas, such as the hippocampus (Zhang et al., 2018; Lloyd et al., 2019). Microglial function is like peripheral macrophages and there has been described phenotypic changes against injury detection. Some authors divide microglia into a classical proinflammatory state (M1) and an alternative anti-inflammatory state (M2) (Wang et al., 2018). Microglia cells undergo an inflammatory polarization phenotype in response to injuries, such as pathogens or tissue damage. This phenotype is characterized by increased pro-inflammatory cytokines production, such as interleukin$1 \beta$ (IL-1 $\beta$ ) or tumor necrosis factor- $\alpha$ (TNF- $\alpha$ ) and enhanced immune responses, including cellular recruitment through chemotaxis and phagocytosis (Subhramanyam et al., 2019; Leng and Edison, 2020). As a result, activated immune cells are recruited to the inflammatory site to remove the injury (Subhramanyam et al., 2019). However, this process must be tightly regulated because uncontrolled or excessive inflammation can lead to tissue damage (Baik et al., 2019). Microglial cells are the first line of defense, as they can destroy or eliminate different pathogens by secreting pro-inflammatory factors/cytotoxic mediators or through their phagocytic function (Zhang et al., 2018; Akhmetzyanova et al., 2019). Nevertheless, an extra accumulation of these mediators caused by microglia chronic activation can also improve neuronal damage and may increase post stroke symptoms (Wang et al., 2018). This statement has been confirmed by post-mortem analysis of brain chronic disorders, such as neurodegenerative diseases like AD (Forloni and Balducci, 2018; Sierksma et al., 2020). Microglia are involved in tissue repair, debris removal, and the maintenance of normal tissue dynamics after infection or injury, especially in the M2 polarized state. The resident microglia are M2 polarized during the early stages of stroke; however, they are transformed into the M1 polarized state in the ischemic penumbra region (Wang et al., 2018). Preclinical data indicate that anti-inflammatory therapy may be effective for stroke or $\mathrm{AD}$, where the strategy is to modulate immune cell function by proinflammatory cytokine release suppression and enhancing anti-inflammatory cytokine expression (Sun et al., 2020).

\section{Phenotype M1 or M2 as an Indicator}

Based on the injury and stimuli that induces microglial cells activation, these cells polarize into phenotypes or subtypes: the pro-inflammatory M1 phenotype, or the anti-inflammatory and immunosuppressive M2 phenotype (Forloni and Balducci, 2018; Song and Li, 2018; Lauro and Limatola, 2020). Phenotype M1 is induced by classical activation, where microglia secrete a high level of pro-inflammatory factors including Interleukin-1 $\beta$ (IL$1 \beta$ ), Interleukin-6 (IL- 6) and TNF- $\alpha$, with high production of nitric oxide (NO) and ROS, which can cause damage to the surrounding neuronal cells (Hickman et al., 2018; Zhang, 2019), TNF- $\alpha$ can also induce an increase in the expression of glutaminase and Connexin-32, which increases the release of glutamate from microglia and enhances the excitotoxicity associated with neuronal damage (Takeuchi et al., 2006). Toll-like receptors (TLRs) have a fundamental role in innate immunity, and they can be activated by different molecules from pathogens called pathogen-associated molecular patterns (PAMPs) (Zolezzi and Inestrosa, 2017; Lauro and Limatola, 2020). This interaction between TLRs and PAMPs activates resident cells to release proinflammatory cytokines. TLR4 is highly expressed in microglia and TLR4-dependent microglial activation has been described on neurodegenerative diseases like AD or after stroke (Rojo et al., 2014; Tang and Le, 2015; Subhramanyam et al., 2019; Kolosowska et al., 2020). Additionally, TLR4 is also responsible for chronic neuroinflammation leading to brain damage after stroke, as it induces the production and release of TNF- $\alpha$, IL-6, and NO, causing neuronal cell death (Bougarne et al., 2018; Song and Li, 2018; Lauro and Limatola, 2020). Otherwise, M2 phenotype is induced by a different mechanism of microglial activation (Liu et al., 2020). M2 microglia are 
the major effector cells with the potential to counteract proinflammatory reactions and promote repair genes expression. Moreover, after the classical activation, an anti-inflammatory and repair phase is initiated to achieve tissue homeostasis (Rojo et al., 2014). The anti-inflammatory cytokines IL-4, IL-13, IL10 , and TGF- $\beta$ are the most secreted molecules by M2 subtype microglia to balance the pro-inflammatory responses. Thus, these activated cells secrete several factors for tissue repair and extracellular matrix remodeling (Zolezzi and Inestrosa, 2017). M2 polarization induces activation of the transcription factor (NFKB -p50) that is associated with the inhibition of M1activation genes (Tang and Le, 2015). Secretion of IL-4, IL-10, and TGF- $\beta$ by M2-activated microglia, promote innate immune responses, down-regulate M1-mediated reactions, and inhibit inflammatory functions. IL-10 is characterized as a cytokine that regulates pro-inflammatory response. Pre-treatments with IL10 decrease nuclear translocation of the p50 and p65 subunits of NF- $\mathrm{KB}$ and production of several proinflammatory cytokines as IL- 6 or TNF- $\alpha$. Furthermore, IL-10 exerts neuroprotective effects and a diminished IL-10 level is associated with increased stroke risk (Sun et al., 2020). Nevertheless, the two microglia activated phenotypes mentioned, could transition into each other in different contexts that may contribute to pathogenic forms of neuroinflammation in chronic situations such as neurodegenerative diseases (Parada et al., 2015; Yang et al., 2017; Zhang et al., 2018; Castillo et al., 2019; Zhang, 2019; dos Santos et al., 2021).

In $\mathrm{AD}$, microglia surrounding the plaques to phagocyte $\mathrm{A} \beta$ generally manifest M2 activation phenotype and this phagocytic activity of microglia is attenuated by pro-inflammatory cytokines such as IFN- $\gamma$, IL-1 $\beta$, and TNF- $\alpha$, which are mainly secreted by M1 microglia (Yang et al., 2017). Furthermore, the M2 phenotype is maintained at old ages of transgenic mice models, suggesting that activated microglia surrounding $A \beta$ plaques adopted an alternative phenotype (Tang and Le, 2015). In 18month-old mice, microglial activation is detected in hippocampal areas free of plaques, exhibiting M1 phenotypes that produce neurotoxic results (Hickman et al., 2018). Otherwise, after stroke, microglial cells are the first cell type to react, and they are activated by multiple cytokines and plasma proteins. Microglia adjacent to necrotic tissue and their peripheral regions at the beginning of a stroke are M2 type, phagocytizing and removing cell debris, necrotic tissue, and toxic metabolites (Hickman et al., 2018; Mancuso et al., 2019a,b). After approximately 24 h, M2 microglia are superseded by M1 subtypes, which release additional pro-inflammatory cytokines and exacerbate neuronal cell damage (Zhou et al., 2020). Finally, they release proinflammatory cytokines, chemokines, and neurotoxic factors, including IL-1 $\beta$, IL-6, TNF- $\alpha$, and NO that induce neurotoxicity (Wright et al., 2013; Wójtowicz et al., 2020). Related to this mechanism is the progression of many brain disorders, such as ischemic stroke and neurodegenerative diseases, where the microglia are chronically activated, amplifying the death of neurons. Also, studies have found that the expression of type M2 polarization receptors and IL-4 secretion decreased with age (Wang et al., 2018). Hence, suppression of the microgliamediated neuroinflammation is a potential therapeutic strategy to treat these brain disorders or prevent the cognitive impairment associated with them. Recently the concepts of homeostatic and reactive microglia have been described and related to major $\mathrm{AD}$ risk factors (such as age and sex), linking to a multifunctional microglial response to $A \beta$ plaques that evolves a continuous spectrum of microglial molecular phenotypes (Mancuso et al., 2019b; Sala Frigerio et al., 2019).

Besides, microglial activation into a pro-inflammatory or anti- inflammatory phenotype are driven by factors released from injured cells called damage-associated molecular patterns (DAMPs) or PAMPs, nonetheless, receptors and intracellular pathways involved are poorly understood. Several DAMPs are released in the brain during the inflammatory process such as misfolding proteins, nucleic acids, or nucleotides, mainly ATP (Di Virgilio and Sarti, 2018). Furthermore, the increase of extracellular ATP is recognized as a cell injury signal and pro-inflammatory stimulus. Microglial cells express receptors for extracellular ADP/ATP nucleotides denominated purinergic $\mathrm{P} 2$ receptors (P2Rs). There are two types of P2R: metabotropic P2YRs and ionotropic P2XRs (Suurväli et al., 2017).

\section{Association Between Purinergic Receptors and Microglial Activation}

Purinergic receptors are activated by purines and are divided into two major families: the P1 or adenosine receptors and P2Y and P2X receptors activated by ADP/ATP (Burnstock, 2018). The first two types are $G$ protein-coupled receptors, whereas P2X are ligand-gated ion channel receptors (Sáez-Orellana et al., 2015). To date eight P2Y receptors have been described in humans: P2Y1, P2Y2, P2Y4, P2Y6, P2Y11, P2Y12, P2Y13, and P2Y14 (Jacobson et al., 2020). These receptors are subdivided in two groups according to the primary $\mathrm{G}$ protein that they are associated with, P2Y1-like (P2Y1, 2, 4, 6, and 11) are coupled mainly with Gq, while P2Y12-like (P2Y12-14) are coupled with Gi (Jacobson et al., 2020). P2Y receptors are widely expressed in the CNS in all cell types, where they play an important role in glianeuron communication, neurotransmission, and neurogenesis [for a more detailed review of P2Y in CNS please see: (Agostinho et al., 2020; Zarrinmayeh and Territo, 2020)].

In mammals, seven P2X subunits are described (P2X 17). The receptors are conformed by homo- or heterotrimers, with a central pore permeable to $\mathrm{Na}^{+}, \mathrm{K}^{+}$and $\mathrm{Ca}^{2+}$ (Illes et al., 2021). Each subunit is composed of two transmembrane domains, intracellular $\mathrm{N}$ - and C-termini and a large extracellular loop. P2X subunits are widely expressed in CNS in all cell types, but some subunits are expressed only in certain cell types, such as P2X 1-3 in neurons and P2X 7 in glia (Illes et al., 2021). Of interest for us, P2X4R is expressed in neurons and glia, particularly in microglia; and is expressed in amygdala, basal ganglia, cerebellum, cerebral cortex, hindbrain, hippocampus, hypothalamus, midbrain, olfactory bulb, and spinal cord (Duveau et al., 2020). Native and recombinant P2X4R show a rapid activation and a slow and incomplete desensitization, and they have a high sensitivity to ATP (EC50 1-10 $\mu \mathrm{M}$ ) and permeability to $\mathrm{Ca}^{2+}$ (Kaiser et al., 2016). A particular characteristic of this purinergic receptor 
is its highly and constitutively internalization due to the presence of a non-canonic motif YXXG $\Phi$ which is mediated by the $\mu 2$ subunit of the adaptive protein 2 (AP2) and clathrin. This intracellular located P2X4R is functional and plays an important role in the secretion and activation of pulmonary surfactant (Fois et al., 2018), and in the fusion and trafficking of lysosomes (Murrell-Lagnado and Frick, 2019). It has been proposed that the homomeric receptors P2X 2, 4 , and 7 dilate their central pore in response to prolonged stimulation, which could allow the permeation of molecules of up-to $800 \mathrm{Da}$, in the case of P2X 7 in microglia this transition pore would be able to induce cell death and apoptosis (Bernier et al., 2012). Some authors have argued that this process is not due to a pore dilation but rather due to the interaction with associated proteins, especially Pannexin-1 (Panx-1), considering that the use of inhibitors for this channel inhibited the permeability to large cations induced by prolonged ATP exposure (Pelegrin and Surprenant, 2006), however, newer evidence utilizing similar strategies have showed that even in the absence of Pannexin-1 by silencing or Knock out there is still permeation of larger molecules (Qu et al., 2011; Alberto et al., 2013). Some reports propose that there is an immediate permeation to large cations after ATP activation, for instance, single channel studies in P2X7R showed no increase in the amplitud or conductance in the channel during prolonged exposure to ATP (Raouf et al., 2007). More recently, it has been demonstrated that the P2X7R expression in purified lysosome (without any other cellular components) is sufficient to form channels permeable to molecules up to $\sim 900 \mathrm{Da}$ and that lipid membrane constitution may play an important role in the P2X7R pore diameter (Karasawa et al., 2017). Therefore, the P2X7R solo expression seems sufficient to form a pore permeable to large cations upon immediate ATP exposure, nevertheless other mechanisms that allow these big molecules passage, such as Panx-1, are still possible.

The participation of different purinergic receptors in pathological processes has been described, among them the P2Y1, P2Y2, P2Y6, P2Y12, P2X4, and P2X7 receptor (Cieślak and Wojtczak, 2018). For many of these receptors, their upregulation and increase in their activity have been described. Interestingly the upregulation of P2X2, P2X 4 and P2X7 has been reported in the brain of $\mathrm{AD}$ patients (McLarnon et al., 2006; Varma et al., 2009; Godoy et al., 2021). In addition, a recent report has shown $\mathrm{P} 2 \mathrm{X} 4 \mathrm{R}$ and $\mathrm{P} 2 \mathrm{X} 7 \mathrm{R}$ co-expression in human neurons from the frontal cortex with no differences between $\mathrm{AD}$ patients and age-matched control group, suggesting that the increased expression of P2XR in brain observed before, might be occurring in glial cells (Gaff et al., 2021).

The concomitant increase of P2X4R and P2X7R is interesting, because they share a high degree of sequence homology $(45.3 \%$ in rat, $46.7 \%$ in human and $47.3 \%$ in mouse), and they are located in the same chromosome and in close proximity of each other in this organisms, and it is suggested that $\mathrm{P} 2 \mathrm{X} 7$ arose as a gene duplication of P2X4 and therefore the mechanism of increased expression could be similar for both receptors (Loera-Valencia et al., 2015; Hou and Cao, 2016; Suurväli et al., 2017).
Furthermore, in mouse lung epithelial cells the silencing of $\mathrm{P} 2 \mathrm{X} 4 \mathrm{R}$ induces an increased P2X7R expression and, the P2X7R down-regulation improves P2X4R expression (Weinhold et al., 2010). In addition, it has been reported $P 2 X 4 R$ negative mice with increased P2X7R expression with passenger mutations altering the receptor's function (Er-Lukowiak et al., 2020; Ellegaard et al., 2021). Similar results were obtained using a transgenic mouse line that expresses soluble GFP by P2X7R promoter, increasing the expression of P2X4R (Ramírez-Fernández et al., 2020). However, has been reported a concomitant decrease in the expression of P2X4R and 7 in liver and kidney of mice deficient for P2X4R or 7 (Craigie et al., 2013; Besnard et al., 2016). Additionally, both receptors tend to be expressed in similar cell types suggesting that these receptors could have complementary functions and may be overlapped (Suurväli et al., 2017).

In spite of that, several results show an increment of ATP in the extracellular environment in different diseases such as $\mathrm{AD}$ and stroke (Wilkaniec et al., 2017; Ye et al., 2017; Srivastava et al., 2020). This extracellular ATP activates P2X receptors, increasing their activity and triggering toxic effects on neurons and glial cells. P2X receptor subunits are present in pre- and postsynaptic sites (Ye et al., 2017). Thus, presynaptic P2X receptors stimulate glutamate release in sensory neurons, whereby they may control intracellular calcium homeostasis. Notably, P2X4R is mainly expressed in microglial cells and is involved in several functions as pain control, anxiety, and memory (Bertin et al., 2020; Montilla et al., 2020; Inoue and Tsuda, 2021).

\section{Increased Expression of P2X4 Receptor on Microglial Cells}

The first evidence of Purinergic receptors in ischemia was obtained in in vitro experiments by the group of Volontè in Italy. They tested chemically induced ischemia and hypoglycemia, together with wide-range P2 antagonists in cerebellar cultures, where they observed neuroprotection and a decrease in cell death (Cavaliere et al., 2001a,b). Importantly, they also described a marked increase of P2X4R expression (Cavaliere et al., 2002). Furthermore, they analyzed organotypic cultures and in vivo models of ischemia (carotid occlusion in gerbils) and using histological and biochemical analysis they observed an increase of P2X4R only in microglia (Cavaliere et al., 2003). Interestingly, in vitro the application of $\mathrm{P} 2$ agonists mimicked the effects of ischemia, and the use of non-selective antagonists decreased the cellular damage observed (Cavaliere et al., 2005). The increase of $\mathrm{P} 2 \mathrm{X} 4 \mathrm{R}$ in microglia is also observed in models of neuropathic pain (Tsuda et al., 2003), where the application of P2X antagonists and the silencing of $\mathrm{P} 2 \mathrm{X} 4 \mathrm{R}$ reduced the observed allodynia. This increased expression is also observed in other pathological processes, such as spinal cord injury, inflammatory pain, chronic migraine, and pre-term hypoxia-ischemia (Wixey et al., 2009; Toulme et al., 2010; de Rivero Vaccari et al., 2012; Tasca et al., 2015; Matsumura et al., 2016; Long et al., 2018; Su et al., 2019; Bertin et al., 2020; Trang et al., 2020). Otherwise, the overexpression of P2X4R in the hippocampus of a mice model altered Long Term Potentiation (LTP) and plasticity at CA1 synapses (Bertin et al., 2020). These findings may be related to an 
increased P2X4R expression in the hippocampus of $\mathrm{AD}$ patients with severe cognitive impairment, suggesting that upregulated P2X4R may contribute to synaptic dysfunction and microglia phagocytic function (Raouf et al., 2007; Godoy et al., 2019; Han et al., 2020; Nguyen et al., 2020; Trang et al., 2020).

The increased expression of P2X4R is mediated by sequential activation of Interferon Regulatory Factor 8 (IRF8), which is a critical regulator of reactive microglia, that induces the expression of repair genes including IRF5, that in turn binds to the promoter region of the P2rx4 gene inducing its expression (Masuda et al., 2012, 2014). P2X4R up-regulation seems to be associated with the neuroinflammation process, which is also related to microglial activation (Raouf et al., 2007; Han et al., 2020). P2X4R activation drives microglia motility via the phosphatidylinositol-3-kinase (PI3K)/Akt pathway (Ohsawa et al., 2007). Also, these receptors are specifically involved in inflammatory-mediated prostaglandin E2 (PGE2) production, which contributes to pain-related inflammation (Montilla et al., 2020). The activation of this pathway by P2X4R increases the expression and induces the BDNF secretion by microglia (Ulmann et al., 2008; Trang et al., 2009; Tsuda et al., 2009). BDNF in response to ATP, induces a shift in the neuronal Chloride gradient, making GABA and Glycine less hyperpolarizing and in some cases depolarizing (Coull et al., 2005). This shift is produced via TrkB activation and a decrease in the expression of $\mathrm{K}^{+} \mathrm{Cl}^{-}$ cotransporter KCC2 (Rivera et al., 2002; Ulmann et al., 2008; Trang et al., 2009). In AD models it has been observed a similar pattern, with decreased KCC2 expression, increased BDNF and TNF- $\alpha$ (Doshina et al., 2017; Zhou et al., 2021), which could link both pathologies related to aging and neuroinflammation.

Finally, during neuroinflammation, the activated glial cells secrete inflammatory mediators to modulate inflammatory responses. It has been described that the upregulation peak of $\mathrm{P} 2 \mathrm{X} 4 \mathrm{R}$ during ischemic injury in the brain occurs 2 days later and that its inhibition can promote the microglial polarization into a pro-inflammatory phenotype (Suurväli et al., 2017; Di Virgilio and Sarti, 2018). This evidence shows us the P2X4R regulation as a key point to inflammation process prevention. In addition to neurons and microglia, immunochemical studies have shown P2X4 receptor expression in astrocytes from hippocampal regions (Srivastava et al., 2020). More studies to determine their function and role in pathological processes such as neuroinflammation, are needed.

\section{DISCUSSION}

Considering all this data, including the overexpression of P2X4R in diseases characterized by chronic neuroinflammation and activated microglial cells, the role of this purinergic receptor remains to be elucidated in humans. Some recent work using general $\mathrm{KO}$ mice and myeloid specific mice for P2X4R, together with a specific antagonist has shown that there is a

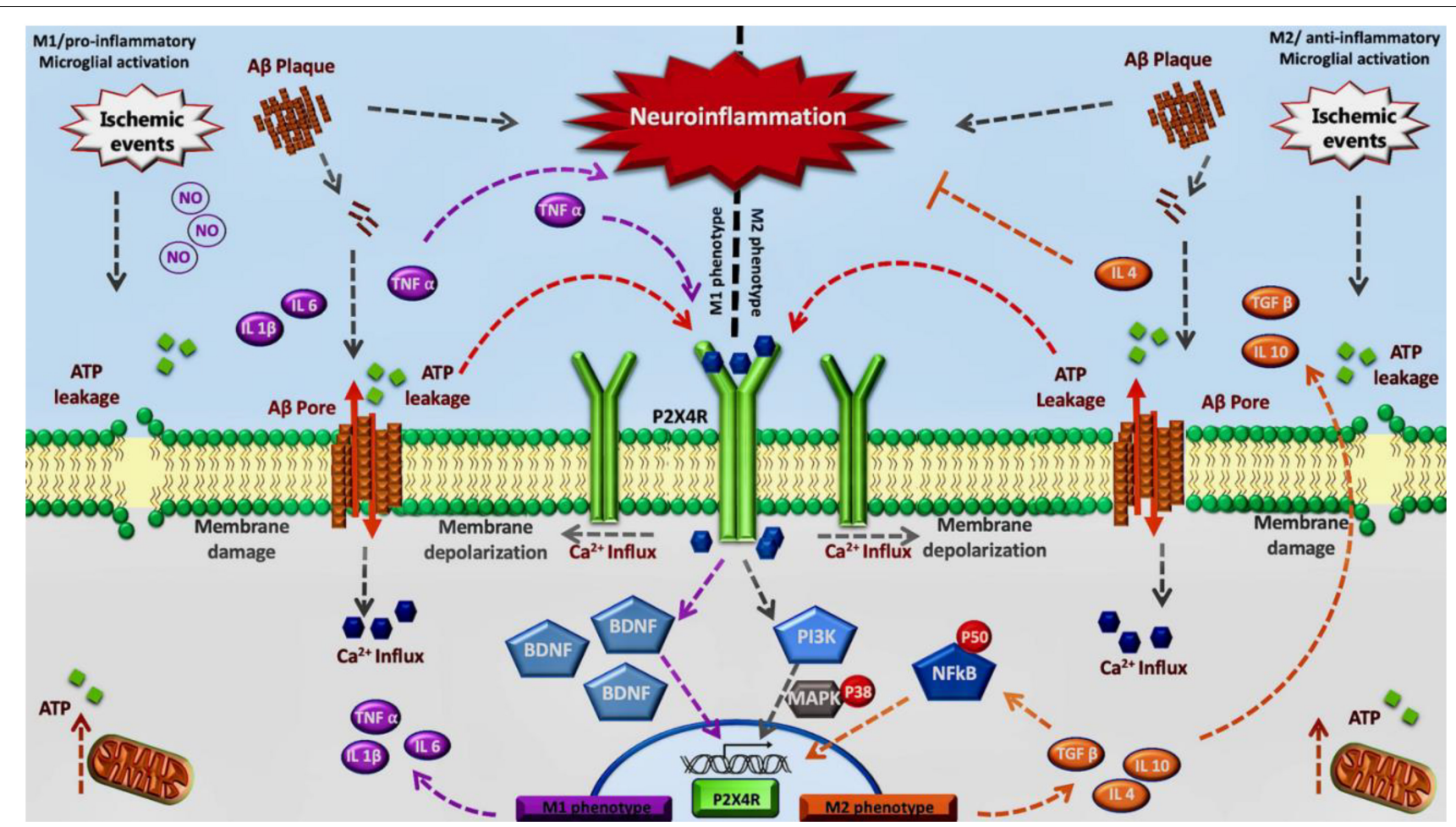

FIGURE 1 | Inhibition of P2X4R in the acute phase could mitigate the effects of M1 transition allowing the appearance of the M2 phenotype: We propose this model considering that in the early stage P2X4 receptors contribute to maintain the M1 phenotype through TNF- $\alpha$ and BDNF signaling. Also, during a final stage, P2X4 may contribute to maintaining M2 phenotype by NFKB pathway. 
critical time window, during the acute phase, where P2X4R inhibition is beneficial for the treatment of stroke, whereas a chronic inhibition could lead to an aggravated depressive state due to the lack of secretion of BDNF (Verma et al., 2017; Srivastava et al., 2020). Therefore, we propose an association between this receptor and the neuroinflammation progress observed in stroke and dementia. We postulate the modulation of P2X4R to regulate a specific change on M1/M2 phenotype of activated microglia observed in neuroinflammation associated with neurodegenerative diseases or stroke. Inhibition of P2X4R in the acute phase could mitigate the effects of M1 transition allowing the appearance of the M2 phenotype (Figure 1).

\section{REFERENCES}

Agostinho, P., Madeira, D., Dias, L., Simões, A. P., Cunha, R. A., and Canas, P. M. (2020). Purinergic signaling orchestrating neuron-glia communication. Pharmacol. Res. 162:105253. doi: 10.1016/J.PHRS.2020.105253

Akhmetzyanova, E., Kletenkov, K., Mukhamedshina, Y., and Rizvanov, A. (2019). Different Approaches to Modulation of Microglia Phenotypes After Spinal Cord Injury. Front. Syst. Neurosci. 13:37. doi: 10.3389/FNSYS.2019.000 37/BIBTEX

Alberto, A. V. P., Faria, R. X., Couto, C. G. C., Ferreira, L. G. B., Souza, C. A. M., Teixeira, P. C. N., et al. (2013). Is pannexin the pore associated with the P2X7 receptor? Naunyn Schmiedebergs Arch. Pharmacol. 386, 775-787. doi: 10.1007/ S00210-013-0868-X/FIGURES/6

Baik, S. H., Kang, S., Lee, W., Choi, H., Chung, S., Kim, J., et al. (2019). A Breakdown in Metabolic Reprogramming Causes Microglia Dysfunction in Alzheimer's Disease. Cell Metabol. 30, 493-507.e6. doi: 10.1016/J.CMET.2019.06.005/ATTACHMENT/218B1465-7137-4FAF8691-039C8039D91F/MMC1.PDF

Bernier, L. P., Ase, A. R., Boué-Grabot, É, and Séguéla, P. (2012). P2X4 receptor channels form large noncytolytic pores in resting and activated microglia. Glia 60, 728-737. doi: 10.1002/GLIA.22301

Bertin, E., Deluc, T., Pilch, K. S., Martinez, A., Pougnet, J. T., Doudnikoff, E., et al. (2020). Increased surface P2X4 receptor regulates anxiety and memory in P2X4 internalization-defective knock-in mice. Mol. Psychiatry 26, 629-644. doi: 10.1038/s41380-019-0641-8

Besnard, A., Gautherot, J., Julien, B., Tebbi, A., Garcin, I., Doignon, I., et al. (2016). The P2X4 purinergic receptor impacts liver regeneration after partial hepatectomy in mice through the regulation of biliary homeostasis. Hepatology 64, 941-953. doi: 10.1002/HEP.28675/SUPPINFO

Bougarne, N., Weyers, B., Desmet, S. J., Deckers, J., Ray, D. W., Staels, B., et al. (2018). Molecular Actions of PPAR $\alpha$ in Lipid Metabolism and Inflammation. Endocr. Rev. 39, 760-802. doi: 10.1210/ER.2018-00064

Burnstock, G. (2018). Purine and purinergic receptors. Brain Neurosci. Adv. 2:2398212818817494. doi: 10.1177/2398212818817494

Castillo, C., Fernández-Mendívil, C., Buendia, I., Saavedra, P., Meza, C., Parra, N. C., et al. (2019). Neuroprotective effects of EpoL against oxidative stress induced by soluble oligomers of $\mathrm{A} \beta$ peptide. Redox Biol. 24:101187. doi: 10. 1016/J.REDOX.2019.101187

Castillo, C., Zaror, S., Gonzalez, M., Hidalgo, A., Burgos, C. F., Cabezas, O. I., et al. (2018). Neuroprotective effect of a new variant of Epo nonhematopoietic against oxidative stress. Redox Biol. 14, 285-294. doi: 10.1016/J.REDOX.2017. 09.010

Cavaliere, F., D’Ambrosi, N., Sancesario, G., Bernardi, G., and Volonté, C. (2001b). Hypoglycaemia-induced cell death: features of neuroprotection by the P2 receptor antagonist basilen blue. Neurochem. Int. 38, 199-207. doi: 10.1016/ S0197-0186(00)00087-5

Cavaliere, F., D’Ambrosi, N., Ciotti, M. T., Mancino, G., Sancesario, G., Bernardi, G., et al. (2001a). Glucose deprivation and chemical hypoxia: neuroprotection by P2 receptor antagonists. Neurochem. Int. 38, 189-197. doi: 10.1016/S01970186(00)00088-7

\section{AUTHOR CONTRIBUTIONS}

CC, FS-O, PAG, and JF contributed to the preparation, revision, and approval of the final manuscript. All authors contributed to the article and approved the submitted version.

\section{FUNDING}

This work was supported by a Chilean National Fund for the Scientific and Technological Development to JF FONDECYT 1200908 and 1161078 .

Cavaliere, F., Dinkel, K., and Reymann, K. (2005). Microglia response and P2 receptor participation in oxygen/glucose deprivation-induced cortical damage. Neuroscience 136, 615-623. doi: 10.1016/J.NEUROSCIENCE.2005.04.038

Cavaliere, F., Florenzano, F., Amadio, S., Fusco, F. R., Viscomi, M. T., D’Ambrosi, N., et al. (2003). Up-regulation of p2x2, p2x4 receptor and ischemic cell death: prevention by p2 antagonists. Neuroscience 120, 85-98. doi: 10.1016/S03064522(03)00228-8

Cavaliere, F., Sancesario, G., Bernardi, G., and Volonté, C. (2002). Extracellular ATP and nerve growth factor intensify hypoglycemia-induced cell death in primary neurons: role of P2 and NGFRp75 receptors. J. Neurochem. 83, 11291138. doi: 10.1046/J.1471-4159.2002.01205.X

Cieślak, M., and Wojtczak, A. (2018). Role of purinergic receptors in the Alzheimer's disease. Purinerg. Signal. 14, 331-344. doi: 10.1007/S11302-0189629-0/TABLES/1

Coull, J. A. M., Beggs, S., Boudreau, D., Boivin, D., Tsuda, M., Inoue, K., et al. (2005). BDNF from microglia causes the shift in neuronal anion gradient underlying neuropathic pain. Nature 438, 1017-1021. doi: 10.1038/nature04223

Craigie, E., Birch, R. E., Unwin, R. J., and Wildman, S. S. (2013). The relationship between P2X4 and P2X7: a physiologically important interaction? Front. Physiol. 4:216. doi: 10.3389/FPHYS.2013.00216/BIBTEX

de Rivero Vaccari, J. P., Bastien, D., Yurcisin, G., Pineau, I., Dalton Dietrich, W., de Koninck, Y., et al. (2012). P2X4 Receptors Influence Inflammasome Activation after Spinal Cord Injury. J. Neurosci. 32, 3058-3066. doi: 10.1523/JNEUROSCI. 4930- 11.2012

Delgado, C., Donoso, A., Orellana, P., Vásquez, C., Díaz, V., and Behrens, M. I. (2010). Frequency and Determinants of Poststroke Cognitive Impairment at Three and Twelve Months in Chile. Dement. Geriatr. Cogn. Disord. 29, 397-405. doi: 10.1159/000305097

dos Santos, I., DIas, M., and Gomes-Leal, W. (2021). Microglial activation and adult neurogenesis after brain stroke. Neural Regener. Res. 16:456. doi: 10.4103/16735374.291383

Doshina, A., Gourgue, F., Onizuka, M., Opsomer, R., Wang, P., Ando, K., et al. (2017). Cortical cells reveal APP as a new player in the regulation of GABAergic neurotransmission. Sci. Rep. 7, 370. doi: 10.1038/s41598-017-00325-2

Duveau, A., Bertin, E., and Boué-Grabot, E. (2020). Implication of Neuronal Versus Microglial P2X4 Receptors in Central Nervous System Disorders. Neurosci. Bull. 36, 1327-1343. doi: 10.1007/S12264-020-00570-Y/FIGURES/3

Ellegaard, M., Hegner, T., Ding, M., Ulmann, L., and Jørgensen, N. R. (2021). Bone phenotype of P2X4 receptor knockout mice: implication of a $\mathrm{P} 2 \mathrm{X} 7$ receptor mutation? Purinerg. Signal. 17, 241-246. doi: 10.1007/S11302-021-09784-9/ FIGURES/2

Er-Lukowiak, M., Duan, Y., Rassendren, F., Ulmann, L., Nicke, A., Ufer, F., et al. (2020). A P2rx7 Passenger Mutation Affects the Vitality and Function of T cells in Congenic Mice. Iscience 23:101870. doi: 10.1016/J.ISCI.2020.101870

Fois, G., Winkelmann, V. E., Bareis, L., Staudenmaier, L., Hecht, E., Ziller, C., et al. (2018). ATP is stored in lamellar bodies to activate vesicular P2X4 in an autocrine fashion upon exocytosis. J. Gener. Physiol. 150, 277-291. doi: 10.1085/JGP.201711870

Forloni, G., and Balducci, C. (2018). Alzheimer's Disease, Oligomers, and Inflammation. J. Alzheimers Dis. 62, 1261-1276. doi: 10.3233/JAD-170819 
Gaff, J., Jackaman, C., Papadimitriou, J., Waters, S., Mclean, C., and Price, P. (2021). Immunohistochemical evidence of P2X7R, P2X4R and CaMKK2 in pyramidal neurons of frontal cortex does not align with Alzheimer's disease. Exp. Mol. Pathol. 120:104636. doi: 10.1016/j.yexmp.2021.104636

Godoy, P. A., Mennickent, D., Cuchillo-Ibáñez, I., Ramírez-Molina, O., SilvaGrecchi, T., Panes-Fernández, J., et al. (2021). Increased P $2 \times 2$ receptors induced by amyloid- $\beta$ peptide participates in the neurotoxicity in alzheimer's disease. Biomed. Pharmacother. 142:111968. doi: 10.1016/J.BIOPHA.2021. 111968

Godoy, P. A., Ramírez-Molina, O., and Fuentealba, J. (2019). Exploring the role of P2X receptors in Alzheimer's disease. Front. Pharmacol. 10:1330. doi: 10.3389/ FPHAR.2019.01330/BIBTEX

Goulay, R., Mena Romo, L., Hol, E. M., and Dijkhuizen, R. M. (2020). From Stroke to Dementia: a Comprehensive Review Exposing Tight Interactions Between Stroke and Amyloid- $\beta$ Formation. Transl. Stroke Res. 11, 601-614. doi: 10.1007/S12975-019-00755-2/FIGURES/1

Hachinski, V., Einhäupl, K., Ganten, D., Alladi, S., Brayne, C., Stephan, B. C. M., et al. (2019). Preventing dementia by preventing stroke: the Berlin Manifesto. Alzheimers Dement. 15, 961-984. doi: 10.1016/J.JALZ.2019.06.001

Han, S. J., Lovaszi, M., Kim, M., D’Agati, V., Haskó, G., and Lee, H. T. (2020). P2X4 receptor exacerbates ischemic AKI and induces renal proximal tubular NLRP3 inflammasome signaling. FASEB J. 34, 5465-5482. doi: 10.1096/FJ.201903287R

Hickman, S., Izzy, S., Sen, P., Morsett, L., and el Khoury, J. (2018). Microglia in neurodegeneration. Nat. Neurosci. 21, 1359-1369. doi: 10.1038/s41593-0180242-x

Hou, Z., and Cao, J. (2016). Comparative study of the P2X gene family in animals and plants. Purinerg. Signal. 12, 269-281. doi: 10.1007/S11302-016-9501-Z/ FIGURES/4

Iadecola, C. (2013). The Pathobiology of Vascular Dementia. Neuron 80, 844-866. doi: 10.1016/j.neuron.2013.10.008

Illes, P., Müller, C. E., Jacobson, K. A., Grutter, T., Nicke, A., Fountain, S. J., et al. (2021). Update of P2X receptor properties and their pharmacology: iUPHAR Review 30. Br. J. Pharmacol. 178, 489-514. doi: 10.1111/BPH.15299

Inoue, K., and Tsuda, M. (2021). Nociceptive signaling mediated by P2X3, P2X4 and P2X7 receptors. Biochem. Pharmacol. 187:114309. doi: 10.1016/J.BCP.2020. 114309

Jacobson, K. A., Delicado, E. G., Gachet, C., Kennedy, C., von Kügelgen, I., Li, B., et al. (2020). Update of P2Y receptor pharmacology: iUPHAR Review 27. Br. J. Pharmacol. 177, 2413-2433. doi: 10.1111/BPH.15005

Jayaraj, R. L., Azimullah, S., Beiram, R., Jalal, F. Y., and Rosenberg, G. A. (2019). Neuroinflammation: friend and foe for ischemic stroke. J. Neuroinflamm. 16:142. doi: 10.1186/S12974-019-1516-2

Kaiser, M., Penk, A., Franke, H., Krügel, U., Nörenberg, W., Huster, D., et al. (2016). Lack of functional P2X7 receptor aggravates brain edema development after middle cerebral artery occlusion. Purinerg. Signal. 12, 453-463. doi: 10.1007/ S11302-016-9511-X/FIGURES/5

Kalaria, R. N. (2018). The pathology and pathophysiology of vascular dementia. Neuropharmacology 134, 226-239. doi: 10.1016/j.neuropharm.2017.12.030

Karasawa, A., Michalski, K., Mikhelzon, P., and Kawate, T. (2017). The P2X7 receptor forms a dye-permeable pore independent of its intracellular domain but dependent on membrane lipid composition. Elife 6:e31186. doi: 10.7554/ eLife.31186.001

Kluge, M. G., Jones, K., Kooi Ong, L., Gowing, E. K., Nilsson, M., Clarkson, A. N., et al. (2018). Age-dependent Disturbances of Neuronal and Glial Protein Expression Profiles in Areas of Secondary Neurodegeneration Post-stroke. Neuroscience 393, 185-195. doi: 10.1016/J.NEUROSCIENCE.2018.07.034

Kolosowska, N., Gotkiewicz, M., Dhungana, H., Giudice, L., Giugno, R., Box, D., et al. (2020). Intracerebral overexpression of miR-669c is protective in mouse ischemic stroke model by targeting MyD88 and inducing alternative microglial/macrophage activation. J. Neuroinflamm. 17, 1-21. doi: 10.1186/ S12974-020-01870-W/FIGURES/1

Lauro, C., and Limatola, C. (2020). Metabolic Reprograming of Microglia in the Regulation of the Innate Inflammatory Response. Front. Immunol. 11:493. doi: 10.3389/FIMMU.2020.00493/BIBTEX

Lavados, P. M., Hoffmeister, L., Moraga, A. M., Vejar, A., Vidal, C., Gajardo, C., et al. (2021). Incidence, risk factors, prognosis, and healthrelated quality of life after stroke in a low-resource community in Chile (NANDU): a prospective population-based study. Lancet Global
Health 9, e340-e351. doi: 10.1016/S2214-109X(20)30470-8/ATTACHMENT/ 74637A4B-25F1-4B39-B551-F781D7A30B81/MMC1.PDF

Lavados, P. M., Sacks, C., Prina, L., Escobar, A., Tossi, C., Araya, F., et al. (2005). Incidence, 30-day case-fatality rate, and prognosis of stroke in Iquique, Chile: a 2-year community-based prospective study (PISCIS project). Lancet 365, 2206-2215. doi: 10.1016/S0140-6736(05)66779-7

Leng, F., and Edison, P. (2020). Neuroinflammation and microglial activation in Alzheimer disease: where do we go from here? Nat. Rev. Neurol. 17, 157-172. doi: 10.1038/s41582-020-00435-y

Lindsay, M. P., Norrving, B., Sacco, R. L., Brainin, M., Hacke, W., Martins, S., et al. (2019). World Stroke Organization (WSO): global Stroke Fact Sheet 2019. Int. J. Stroke 14, 806-817. doi: 10.1177/1747493019881353

Liu, M., Xu, Z., Wang, L., Zhang, L., Liu, Y., Cao, J., et al. (2020). Cottonseed oil alleviates ischemic stroke injury by inhibiting the inflammatory activation of microglia and astrocyte. J. Neuroinflamm. 17, 1-15. doi: 10.1186/S12974-02001946-7/FIGURES/8

Lloyd, A. F., Davies, C. L., Holloway, R. K., Labrak, Y., Ireland, G., Carradori, D., et al. (2019). Central nervous system regeneration is driven by microglia necroptosis and repopulation. Nat. Neurosci. 22, 1046-1052. doi: 10.1038/ s41593-019-0418-z

Loera-Valencia, R., Jaramillo-Polanco, J. O., Linan-Rico, A., Pescador, M. G. N., Bremont, J. F. J., and Barajas-López, C. (2015). Genomic Organization of Purinergic P2X Receptors. Pharmacol. Pharmacy 6, 341-362. doi: 10.4236/PP. 2015.68036

Long, T., He, W., Pan, Q., Zhang, S., Zhang, Y., Liu, C., et al. (2018). Microglia P2X4 receptor contributes to central sensitization following recurrent nitroglycerin stimulation. J. Neuroinflamm. 15, 1-11. doi: 10.1186/S12974-018-1285-3/ FIGURES/6

Lui, S. K., and Nguyen, M. H. (2018). Elderly Stroke Rehabilitation: overcoming the Complications and Its Associated Challenges. Curr. Gerontol. Geriatr. Res. 2018:9853837. doi: 10.1155/2018/9853837

Mancuso, R., van den Daele, J., Fattorelli, N., Wolfs, L., Balusu, S., Burton, O., et al. (2019b). Stem-cell-derived human microglia transplanted in mouse brain to study human disease. Nat. Neurosci. 22, 2111-2116. doi: 10.1038/s41593-0190525- $\mathrm{x}$

Mancuso, R., Fryatt, G., Cleal, M., Obst, J., Pipi, E., Monzón-Sandoval, J., et al. (2019a). CSF1R inhibitor JNJ-40346527 attenuates microglial proliferation and neurodegeneration in P301S mice. Brain 142, 3243-3264. doi: 10.1093/BRAIN/ AWZ241

Masuda, T., Iwamoto, S., Yoshinaga, R., Tozaki-Saitoh, H., Nishiyama, A., Mak, T. W., et al. (2014). Transcription factor IRF5 drives P2X4R+-reactive microglia gating neuropathic pain. Nat. Commun. 5, 3771. doi: 10.1038/ncomms4 771

Masuda, T., Tsuda, M., Yoshinaga, R., Tozaki-Saitoh, H., Ozato, K., Tamura, T., et al. (2012). IRF8 Is a Critical Transcription Factor for Transforming Microglia into a Reactive Phenotype. Cell Rep. 1, 334-340. doi: 10.1016/J.CELREP.2012. 02.014

Matsumura, Y., Yamashita, T., Sasaki, A., Nakata, E., Kohno, K., Masuda, T., et al. (2016). A novel P2X4 receptor-selective antagonist produces anti-allodynic effect in a mouse model of herpetic pain. Sci. Rep. 6:32461. doi: 10.1038/ srep32461

McLarnon, J. G., Ryu, J. K., Walker, D. G., and Choi, H. B. (2006). Upregulated Expression of Purinergic P2X7 Receptor in Alzheimer Disease and Amyloid$\beta$ Peptide-Treated Microglia and in Peptide-Injected Rat Hippocampus. J. Neuropathol. Exp. Neurol. 65, 1090-1097. doi: 10.1097/01.JNEN.0000240470. 97295.D3

Mijajlović, M. D., Pavlović, A., Brainin, M., Heiss, W. D., Quinn, T. J., Ihle-Hansen, H. B., et al. (2017). Post-stroke dementia - a comprehensive review. BMC Med. 15:11. doi: 10.1186/S12916-017-0779-7

Montilla, A., Mata, G. P., Matute, C., and Domercq, M. (2020). Contribution of P2X4 Receptors to CNS Function and Pathophysiology. Int. J. Mol. Sci. 21:5562. doi: 10.3390/IJMS21155562

Murrell-Lagnado, R. D., and Frick, M. (2019). P2X4 and lysosome fusion. Curr. Opin. Pharmacol. 47, 126-132. doi: 10.1016/J.COPH.2019.03.002

Nguyen, H. M., di Lucente, J., Chen, Y. J., Cui, Y., Ibrahim, R. H., Pennington, M. W., et al. (2020). Biophysical basis for Kv1.3 regulation of membrane potential changes induced by P2X4-mediated calcium entry in microglia. Glia 68, 2377-2394. doi: 10.1002/GLIA.23847 
Ohsawa, K., Irino, Y., Nakamura, Y., Akazawa, C., Inoue, K., and Kohsaka, S. (2007). Involvement of P2X4 and P2Y12 receptors in ATP-induced microglial chemotaxis. Glia 55, 604-616. doi: 10.1002/GLIA.20489

Parada, E., Buendia, I., Navarro, E., Avendaño, C., Egea, J., and López, M. G. (2015). Microglial HO-1 induction by curcumin provides antioxidant, antineuroinflammatory, and glioprotective effects. Mol. Nutr. Food Res. 59, 1690-1700. doi: 10.1002/MNFR.201500279

Pelegrin, P., and Surprenant, A. (2006). Pannexin-1 mediates large pore formation and interleukin- $1 \beta$ release by the $\mathrm{ATP}$-gated $\mathrm{P} 2 \mathrm{X} 7$ receptor. EMBO J. 25, 5071-5082. doi: 10.1038/SJ.EMBOJ.7601378

Phipps, M. S., and Cronin, C. A. (2020). Management of acute ischemic stroke. BMJ 368:16983. doi: 10.1136/BMJ.L6983

Qu, Y., Misaghi, S., Newton, K., Gilmour, L. L., Louie, S., Cupp, J. E., et al. (2011). Pannexin-1 Is Required for ATP Release during Apoptosis but Not for Inflammasome Activation. J. Immunol. 186, 6553-6561. doi: 10.4049/ JIMMUNOL.1100478

Ramírez-Fernández, A., Urbina-Treviño, L., Conte, G., Alves, M., Rissiek, B., Durner, A., et al. (2020). Deviant reporter expression and P2X4 passenger gene overexpression in the soluble EGFP BAC transgenic $\mathrm{P} 2 \mathrm{X} 7$ reporter mouse model. Sci. Rep. 10:19876. doi: 10.1038/s41598-020-76428-0

Randolph, S. A. (2016). Ischemic Stroke. Workplace Health Safety 64:444. doi: $10.1177 / 2165079916665400$

Raouf, R., Chabot-Doré, A. J., Ase, A. R., Blais, D., and Séguéla, P. (2007). Differential regulation of microglial P2X4 and P2X7 ATP receptors following LPS-induced activation. Neuropharmacology 53, 496-504. doi: 10.1016/J. NEUROPHARM.2007.06.010

Rivera, C., Li, H., Thomas-Crusells, J., Lahtinen, H., Viitanen, T., Nanobashvili, A., et al. (2002). BDNF-induced TrkB activation down-regulates the $\mathrm{K}^{+}-\mathrm{Cl}^{-}$ cotransporter KCC2 and impairs neuronal $\mathrm{Cl}^{-}$extrusion. J. Cell Biol. 159, 747-752. doi: 10.1083/JCB.200209011

Rojo, A. I., McBean, G., Cindric, M., Egea, J., López, M. G., Rada, P., et al. (2014). Redox control of microglial function: molecular mechanisms and functional significance. Antioxid. Redox Signal. 21, 1766-1801. doi: 10.1089/ARS.2013. 5745/ASSET/IMAGES/LARGE/FIGURE15.JPEG

Sáez-Orellana, F., Godoy, P. A., Silva-Grecchi, T., Barra, K. M., and Fuentealba, J. (2015). Modulation of the neuronal network activity by P2X receptors and their involvement in neurological disorders. Pharmacol. Res. 101, 109-115. doi: 10.1016/J.PHRS.2015.06.009

Sala Frigerio, C., Wolfs, L., Fattorelli, N., Thrupp, N., Voytyuk, I., Schmidt, I., et al. (2019). The Major Risk Factors for Alzheimer's Disease: age, Sex, and Genes Modulate the Microglia Response to A $\beta$ Plaques. Cell Rep. 27, 1293-1306.e6. doi: 10.1016/J.CELREP.2019.03.099/ATTACHMENT/04BF9B46-7C1F-432EA1C7-CE30134A78E2/MMC1.PDF

Sierksma, A., Lu, A., Mancuso, R., Fattorelli, N., Thrupp, N., Salta, E., et al. (2020). Novel Alzheimer risk genes determine the microglia response to amyloid- $\beta$ but not to TAU pathology. EMBO Mol. Med. 12:e10606. doi: 10.15252/EMMM. 201910606

Song, N., and Li, T. (2018). Regulation of NLRP3 inflammasome by phosphorylation. Front. Immunol. 9:2305. doi: 10.3389/FIMMU.2018.02305/ BIBTEX

Srivastava, P., Cronin, C. G., Scranton, V. L., Jacobson, K. A., Liang, B. T., and Verma, R. (2020). Neuroprotective and neuro-rehabilitative effects of acute purinergic receptor P2X4 (P2X4R) blockade after ischemic stroke. Exp. Neurol. 329:113308. doi: 10.1016/J.EXPNEUROL.2020.113308

Su, W. F., Wu, F., Jin, Z. H., Gu, Y., Chen, Y. T., Fei, Y., et al. (2019). Overexpression of P2X4 receptor in Schwann cells promotes motor and sensory functional recovery and remyelination via BDNF secretion after nerve injury. Glia 67, 78-90. doi: 10.1002/GLIA.23527

Subhramanyam, C. S., Wang, C., Hu, Q., and Dheen, S. T. (2019). Microgliamediated neuroinflammation in neurodegenerative diseases. Sem. Cell Dev. Biol. 94, 112-120. doi: 10.1016/J.SEMCDB.2019.05.004

Sun, P., Zhou, W., Yue, H., Zhang, C., Ou, Y., Yang, Z., et al. (2020). Compound AD110 Acts as Therapeutic Management for Alzheimer's Disease and Stroke in Mouse and Rat Models. ACS Chem. Neurosci. 11, 929-938. doi: 10.1021/ ACSCHEMNEURO.9B00651/SUPPL_FILE/CN9B00651_SI_001.PDF

Suurväli, J., Boudinot, P., Kanellopoulos, J., and Rüütel Boudinot, S. (2017). P2X4: a fast and sensitive purinergic receptor. Biomed. J. 40, 245-256. doi: 10.1016/J. BJ.2017.06.010
Takeuchi, H., Jin, S., Wang, J., Zhang, G., Kawanokuchi, J., Kuno, R., et al. (2006). Tumor Necrosis Factor- $\alpha$ Induces Neurotoxicity via Glutamate Release from Hemichannels of Activated Microglia in an Autocrine Manner. J. Biol. Chem. 281, 21362-21368. doi: 10.1074/JBC.M600504200

Tang, Y., and Le, W. (2015). Differential Roles of M1 and M2 Microglia in Neurodegenerative Diseases. Mol. Neurobiol. 53, 1181-1194. doi: 10.1007/ S12035-014-9070-5

Tasca, C. I., Dal-Cim, T., and Cimarosti, H. (2015). In Vitro Oxygen-Glucose Deprivation to Study Ischemic Cell Death. Methods Mol. Biol. 1254, 197-210. doi: 10.1007/978-1-4939-2152-2_15

Toulme, E., Garcia, A., Samways, D., Egan, T. M., Carson, M. J., and Khakh, B. S. (2010). P2X4 receptors in activated C8-B4 cells of cerebellar microglial origin. J. General Physiol. 135, 333-353. doi: 10.1085/JGP.200910336

Trang, M., Schmalzing, G., Müller, C. E., and Markwardt, F. (2020). Dissection of P2X4 and P2X7 Receptor Current Components in BV-2 Microglia. Int. J. Mol. Sci. 21:8489. doi: 10.3390/IJMS21228489

Trang, T., Beggs, S., Wan, X., and Salter, M. W. (2009). P2X4-Receptor-Mediated Synthesis and Release of Brain-Derived Neurotrophic Factor in Microglia Is Dependent on Calcium and p38-Mitogen-Activated Protein Kinase Activation. J. Neurosci. 29, 3518-3528. doi: 10.1523/JNEUROSCI.5714-08.2009

Trejo-Lopez, J. A., Anthony, Yachnis, T., and Prokop, S. (2021). Neuropathology of Alzheimer's Disease. Neurotherapeutics Epub online ahead of print. doi: 10.1007/S13311-021-01146-Y

Tsuda, M., Shigemoto-Mogami, Y., Koizumi, S., Mizokoshi, A., Kohsaka, S., Salter, M. W., et al. (2003). P2X4 receptors induced in spinal microglia gate tactile allodynia after nerve injury. Nature 424, 778-783. doi: 10.1038/nature01786

Tsuda, M., Toyomitsu, E., Kometani, M., Tozaki-Saitoh, H., and Inoue, K. (2009). Mechanisms underlying fibronectin-induced up-regulation of P2X4R expression in microglia: distinct roles of PI3K-Akt and MEK-ERK signalling pathways. J. Cell. Mol. Med. 13, 3251-3259. doi: 10.1111/J.1582-4934.2009. 00719.X

Ulmann, L., Hatcher, J. P., Hughes, J. P., Chaumont, S., Green, P. J., Conquet, F., et al. (2008). Up-Regulation of P2X4 Receptors in Spinal Microglia after Peripheral Nerve Injury Mediates BDNF Release and Neuropathic Pain. J. Neurosci. 28, 11263-11268. doi: 10.1523/JNEUROSCI.2308-08. 2008

Varma, R., Chai, Y., Troncoso, J., Gu, J., Xing, H., Stojilkovic, S. S., et al. (2009). Amyloid- $\beta$ induces a caspase-mediated cleavage of P2X4 to promote purinotoxicity. Neuro Mol. Med. 11, 63-75. doi: 10.1007/S12017-009-8073-2/ FIGURES/6

Verma, R., Cronin, C. G., Hudobenko, J., Venna, V. R., McCullough, L. D., and Liang, B. T. (2017). Deletion of the P2X4 receptor is neuroprotective acutely, but induces a depressive phenotype during recovery from ischemic stroke. Brain Behav. Immunity 66, 302-312. doi: 10.1016/J.BBI.2017.07.155

Vijayan, M., and Reddy, P. H. (2016). Stroke, Vascular Dementia, and Alzheimer's Disease: molecular Links. J. Alzheimers Dis. 54, 427-443. doi: 10.3233/JAD160527

Vijayan, M., Kumar, S., Bhatti, J. S., and Reddy, P. H. (2017). Molecular Links and Biomarkers of Stroke, Vascular Dementia, and Alzheimer's Disease. Progress Mol. Biol. Transl. Sci. 146, 95-126. doi: 10.1016/BS.PMBTS.2016.12.014

Di Virgilio, F., and Sarti, A. C. (2018). Microglia P2X4 receptors as pharmacological targets for demyelinating diseases. EMBO Mol. Med. 10:e9369. doi: 10.15252/ EMMM.201809369

Wang, J., Xing, H., Wan, L., JIang, X., Wang, C., and Wu, Y. (2018). Treatment targets for M2 microglia polarization in ischemic stroke. Biomed. Pharmacother. 105, 518-525. doi: 10.1016/J.BIOPHA.2018.05.143

Weinhold, K., Krause-Buchholz, U., Rödel, G., Kasper, M., and Barth, K. (2010). Interaction and interrelation of P2X7 and P2X4 receptor complexes in mouse lung epithelial cells. Cell. Mol. Life Sci. 67, 2631-2642. doi: 10.1007/S00018-0100355-1/FIGURES/7

Wilkaniec, A., Gąssowska, M., Czapski, G. A., Cieślik, M., Sulkowski, G., and Adamczyk, A. (2017). P2X7 receptor-pannexin 1 interaction mediates extracellular alpha-synuclein-induced ATP release in neuroblastoma SH-SY5Y cells. Purinerg. Signal. 13, 347-361. doi: 10.1007/S11302-017-9567-2

Wixey, J. A., Reinebrant, H. E., Carty, M. L., and Buller, K. M. (2009). Delayed P2X4R expression after hypoxia-ischemia is associated with microglia in the immature rat brain. J. Neuroimmunol. 212, 35-43. doi: 10.1016/J.JNEUROIM. 2009.04.016 
Wójtowicz, S., Strosznajder, A. K., Jeżyna, M., and Strosznajder, J. B. (2020). The Novel Role of PPAR Alpha in the Brain: promising Target in Therapy of Alzheimer's Disease and Other Neurodegenerative Disorders. Neurochem. Res. 45, 972-988. doi: 10.1007/S11064-020-02 993-5

Wright, A. L., Zinn, R., Hohensinn, B., Konen, L. M., Beynon, S. B., Tan, R. P., et al. (2013). Neuroinflammation and Neuronal Loss Precede A $\beta$ Plaque Deposition in the hAPP-J20 Mouse Model of Alzheimer's Disease. PLoS One 8:e59586. doi: 10.1371/JOURNAL.PONE.0059586

Yang, X., Xu, S., Qian, Y., and Xiao, Q. (2017). Resveratrol regulates microglia M1/M2 polarization via PGC- $1 \alpha$ in conditions of neuroinflammatory injury. Brain Behav Immunity 64, 162-172. doi: 10.1016/J.BBI.2017. 03.003

Ye, X., Shen, T., Hu, J., Zhang, L., Zhang, Y., Bao, L., et al. (2017). Purinergic 2X7 receptor/NLRP3 pathway triggers neuronal apoptosis after ischemic stroke in the mouse. Exp. Neurol. 292, 46-55. doi: 10.1016/J.EXPNEUROL.2017.03.002

Zarrinmayeh, H., and Territo, P. R. (2020). Purinergic Receptors of the Central Nervous System: biology, PET Ligands, and Their Applications. Mol. Imaging 19:1536012120927609. doi: 10.1177/1536012120927609

Zhang, L., Zhang, J., and You, Z. (2018). Switching of the microglial activation phenotype is a possible treatment for depression disorder. Front. Cell. Neurosci. 12:306. doi: 10.3389/FNCEL.2018.00306/BIBTEX

Zhang, S. (2019). Microglial activation after ischaemic stroke. Stroke Vascul. Neurol. 4, 71-74. doi: 10.1136/SVN-2018-000196

Zhao, S. C., Ma, L. S., Chu, Z. H., Xu, H., Wu, W. Q., and Liu, F. (2017). Regulation of microglial activation in stroke. Acta Pharmacol. Sin. 38, 445-458. doi: 10.1038/aps.2016.162
Zhou, F., Liu, R., Han, P., Zhang, X., Li, Z., Zhang, S., et al. (2020). Pertussis Toxin Ameliorates Microglial Activation Associated With Ischemic Stroke. Front. Cell. Neurosci. 14:152. doi: 10.3389/FNCEL.2020.00152/BIBTEX

Zhou, Y., Cheng, Y., Li, Y., Ma, J., Wu, Z., Chen, Y., et al. (2021). Soluble $\beta$-amyloid impaired the BA inhibition by mediating KCC2 in early APP/PS1 mice. BioSci. Trends 15, 330-340. doi: 10.5582/BST.2021.01245

Zolezzi, J. M., and Inestrosa, N. C. (2017). Wnt/TLR dialog in neuroinflammation, relevance in Alzheimer's disease. Frontiers in Immunology 8:187. doi: 10.3389/ FIMMU.2017.00187/BIBTEX

Conflict of Interest: The authors declare that the research was conducted in the absence of any commercial or financial relationships that could be construed as a potential conflict of interest.

Publisher's Note: All claims expressed in this article are solely those of the authors and do not necessarily represent those of their affiliated organizations, or those of the publisher, the editors and the reviewers. Any product that may be evaluated in this article, or claim that may be made by its manufacturer, is not guaranteed or endorsed by the publisher.

Copyright (c) 2022 Castillo, Saez-Orellana, Godoy and Fuentealba. This is an openaccess article distributed under the terms of the Creative Commons Attribution License (CC BY). The use, distribution or reproduction in other forums is permitted, provided the original author(s) and the copyright owner(s) are credited and that the original publication in this journal is cited, in accordance with accepted academic practice. No use, distribution or reproduction is permitted which does not comply with these terms. 